\title{
La didáctica de las matemáticas: Un vistazo con futuros docentes
}

José Eligio Guzmán Contreras ${ }^{1}$

\section{INFORMACIÓN DEL ARTÍCULO}

Recibido: 30.04 .2020

Aprobado: 30.05 .2020

\section{Palabras claves:}

Didáctica,

Enseñanza,

Aprendizaje,

Estrategias.

\section{RESUMEN}

El presente escrito tiene la finalidad de presentar los resultados de la percepción que tienen estudiantes de la Carrera de Física-Matemática de la Facultad Regional Multidisciplinaria de Chontales de la UNAN-Managua, sobre la Didáctica de las Matemáticas; los desafío que los docentes enfrentan en la actualidad, así como las características que presenta un proceso de enseñanza tradicional, sin dejar de señalar cuál o cuáles serían las estrategias innovadoras que motiven o despierten el interés de los estudiantes por las matemáticas. Todo esto basado en sus propias experiencias acumuladas en el transcurso de sus años de estudios, las que han logrado interiorizar sobre la Didáctica de las Matemáticas mediante el compartir con los docentes y, las teorías alrededor del cómo hacer de la asignatura de matemática una clase amigable y hasta cierto punto agradable. Se ha partido de la primicia de que: "si entender las matemáticas es difícil, cuanto más poderla enseñar". Esto induce a una reflexión consciente sobre la importancia del dominio de la didáctica que con mucha regularidad se presentan dificultades al momento de desarrollar tal asignatura, así como las consideraciones para mejorar el proceso de enseñanza-aprendizaje. De igual manera las estrategias innovadoras como el uso de las TIC, que se consideran de gran ayuda para romper el paradigma de que las matemáticas son solo para personas especiales.

\section{Didactics of Mathematics: a look with future teachers}

\begin{abstract}
The purpose of this paper is to present the results of the perception that students have of the Physics-Mathematics Career of the Regional Multidisciplinary Faculty of Chontales of UNANManagua, on the Didactics of Mathematics; the challenges that teachers currently face, as well as the characteristics that a traditional teaching process presents, without ceasing to point out what or which innovative strategies would be that motivate or awaken students' interest in mathematics. All this based on their own experiences accumulated in the course of their years of studies, which they have managed to internalize about Math's Didactics by sharing with teachers
\end{abstract}

\footnotetext{
${ }^{1}$ Doctor en Matemáticas Aplicadas. Profesor de la Universidad Nacional Autónoma de Nicaragua, Managua- Facultad Regional Multidiscipliaria - Chontales. Email: jose.guzman@unan.edu.ni ㅁ: https://orcid.org/oooo-ooo1-7483-4663
} 
and the theories about how to make the subject of mathematics a friendly class, and to a certain extent pleasant. It has started from the scoop that: "if understanding mathematics is difficult, the more you can teach it". This leads to a conscious reflection on the importance of the domain of didactics, which regularly present difficulties when developing such a subject, as well as the considerations to improve the teaching-learning process. In the same way, innovative strategies such as the use of ICTs, which are considered to be of great help to break the paradigm that mathematics is only for special people.

\section{Introducción}

Al hacer referencia sobre la Didáctica, probablemente nos encontremos con la definición o definiciones clásicas, que tienen que ver con el "Arte de Enseñar", sin embargo, cuando nos referimos a la parte específica sobre las matemáticas de forma heurística podemos visualizar sus avances en contraposición de las definiciones primitivas que aseguraban que existía un único método para enseñar cualquier ciencia o letra.

Existen diversos trabajos con la intensión de mejorar el proceso de enseñanzaaprendizaje de las matemáticas, sin embargo, el presente está enmarcado en una reflexión sobre el conocimiento consciente que tienen los estudiantes de cuarto año de la carrera de Física-Matemática de la Facultad Regional Multidisciplinaria de Chontales (FAREM-Chontales), sobre la Didáctica de las Matemáticas, ya que pronto estarán ejerciendo la labor docente. La preocupación radica en que la sociedad demanda cada día una "mejor enseñanza de los números", y se refieren a que los docentes saben mucho para ellos, pero, que no pueden enseñarlas, y que al explicarlas no se les entiende, por tal razón los propósitos orbitan alrededor de realizar algunas propuestas que surgen del seno de los mismos estudiantes apegados al contexto, realidad e intereses de los educandos.

Estas reflexiones quedan a disposición de aquellos docentes que imparten la asignatura de matemática, como una herramienta que les permita enseñar una clase que puedan brindarles a sus estudiantes, y provoquen la satisfacción que sentimos los que hemos decidido dedicar nuestra vida a tan magna labor como es la docencia, sin importar el nivel del sistema educativo y que siempre estamos tratando de renovar e innovar para mejorar el proceso de enseñanza-aprendizaje de los números.

\section{Literatura}

\subsection{Aproximación al concepto de la didáctica fundamental}

Estamos de acuerdo como docentes de matemática que lo didáctico hace referencia a la enseñanza-aprendizaje, aunque este significado ha venido evolucionando en torno al aprendizaje matemático, el enfoque tradicional no lo considera como entidad de estudio para "enseñar matemáticas" o "aprender matemáticas", ya que se pensaba que existe un solo método para enseñar ciencia o letras. 
Sin embargo, la didáctica fundamental hace su aparición en la década de los 70, cuando se hacen las primeras formulaciones de la Teoría de Situaciones Didácticas donde se considera por primera vez la necesidad para la didáctica de utilizar un modelo propio de la actividad matemática, así pues, el objetivo fundamental de la didáctica es el de definir un "conocimiento matemático", mediante una "situación" tal que ésta situación produzca "situaciones a-didácticas" en los alumnos que permitan engendrar una buena representación del conocimiento (Brousseau, 2007).

Aunque parece un juego de palabras la realidad es que se deben crear las condiciones apoyados con las diversas herramientas o medio como se le llama en la teoría de las situaciones didácticas en las que al final el estudiante debe interactuar con la intensión que producto de ese juego, se pueda provocar un aprendizaje.

\subsection{La Didáctica de las Matemáticas}

La didáctica de las matemáticas es una disciplina científica joven, la Comisión Internacional para la Instrucción Matemática (ICMI), se estableció en 1908, que se dedica a identificar y a explicar fenómenos, y a tratar de resolver problemas, ambos relacionados con la enseñanza y el aprendizaje de las matemáticas; estos problemas y fenómenos se pueden manifestar dentro y fuera de la escuela. Para estudiar dichos problemas y fenómenos, la didáctica utiliza teorías y métodos propios, pero también utiliza muchas otras disciplinas importantes como la psicología, la antropología, la ergonomía, la sociología, las ciencias políticas, entre otros.

En la actualidad Martínez y Romero (2019) citando a Godino (2011) quien afirma que la concepción fundamental de la Didáctica de la Matemática como ciencia se interesa por la producción y comunicación de los conocimientos matemáticos, en lo que esta producción y comunicación tienen aspectos específicos similares, tales como:

- Las operaciones esenciales de la difusión de los conocimientos, las condiciones de esta difusión y las transformaciones que produce.

- Las instituciones y las actividades que tienen por objeto facilitar estas operaciones.

Aunque da la impresión de que roza con una concepción distinta a la que inicialmente se pensaba que no era necesaria ya que se pensaba que existe un solo método para las ciencias y las letras, se sigue trabajando por una definición que satisfaga la inquietud de los nuevos docentes.

\subsection{Problemas y dificultades para enseñar las matemáticas}

Se podrían enumerar una serie de dificultades en relación con la enseñanza de las matemáticas, en este sentido, RTVE.ES afirma que se ha tratado de confrontar estos 
resultados del Informe del Programa Internacional para la Evaluación de Estudiantes o informe PISA con la realidad de las aulas. Los profesores de matemáticas consultados admiten que no es fácil lograr que el alumno disfrute de una asignatura que es inevitablemente abstracta y de esas que no se aprueban con un atracón de estudio a última hora, sino con trabajo concienzudo, mucho ensayo y mucho error.

"Hace falta abstracción para las matemáticas, pero si no se logra atraer a los alumnos, perdemos a aquellos a los que les cuesta llegar y también a los que no, que se aburren. Hay que ser sexy para todo, hasta para enseñar matemáticas", afirma con facilidad Clara Grima, doctora en Matemáticas y dedicada desde hace años a la divulgación de la singular belleza de las matemáticas.

Si a todo esto le agregamos que el estudiante no les encuentra sentido a las matemáticas, no despertamos el interés por ellas, y si a eso le asociamos las problemáticas del hogar, la situación sociopolítica, incluso sanitaria como la que en la actualidad se vive como el Covid-19, no encontrará un sentido práctico para poder apreciar la importancia de esta asignatura cuando está en juego la vida misma.

\subsection{Características de un proceso de enseñanza tradicional}

En el sitio Web de la OEI, se hace referencia sobre la Escuela Tradicional que aparece en el siglo XVII en Europa con el surgimiento de la burguesía y como expresión de modernidad. Encuentra su concreción en los siglos XVIII y XIX con el surgimiento de la Escuela Pública en Europa y América Latina, con el éxito de las revoluciones republicanas de doctrina político-social del liberalismo.

En este modelo el contenido viene dado por los conocimientos y valores acumulados por la sociedad y las ciencias, como verdades acabadas, todo lo cual aparece divorciado de las experiencias y realidades del alumno y su contexto, contenidos representados en el maestro. El contenido curricular es racionalista, académico, apegado a la ciencia y se presenta metafísicamente, sin una lógica interna, en partes aisladas, lo que conlleva a desarrollar un pensamiento empírico, no teórico, de tipo descriptivo.

La enseñanza tradicional tiene sus propias características, Van Arken, (2018), las estipulas de forma resumida y sencilla como:

- Rol del docente: Es el centro del proceso de enseñanza y educación. Informa conocimientos acabados (sujeto principal).

- Rol del estudiante: Tiene poco margen para pensar y elaborar conocimientos. Se le exige memorización. No hay un adecuado desarrollo de pensamiento teórico. Tiene un rol pasivo. 
- Características de la clase: Transmisión verbal de gran volumen de información. Objetivo elaborado de forma descriptiva dirigido más a la tarea del profesor, no establece habilidades. No hay experiencias vivenciales. Los contenidos se ofrecen como segmentos fragmentados, desvinculados de la totalidad. Se realizan pocas actividades de carácter práctico por el alumno. No se controla cómo ocurre el proceso de aprendizaje. Se evalúan resultados y a un nivel reproductivo. Método fundamentalmente expositivo. Forma: grupo presencial.

\subsection{Estrategias innovadoras en el proceso de enseñanza-aprendizaje de las matemáticas.}

Actualmente es muy común escuchar en los círculos docentes propuestas que estén referidas a estrategias innovadoras para una mejor enseñanza de las matemáticas, incluso se propone aquellas que apuntan al uso de estrategias didácticas para un aprendizaje constructivista tomando como punto de partida la función que cumple el maestro y el alumno en el proceso de aprendizaje lógico-matemático, determinando el uso de las estrategias didácticas para un aprendizaje constructivista de las matemáticas con el uso de tecnología, juegos, recursos y material didáctico en los estudiantes generando el desarrollo cognitivo a través del razonamiento, la imaginación, la creación y experimentación de cada contenido que se transmite en el aula (Melquiades, 2014).

\section{Metodología de la investigación}

La población involucrada en el estudio son los 27 estudiantes de la Carrera de Física-Matemática, que recibían la clase de Didáctica de las Matemáticas, el estudio es tipo exploratorio, que según Danhke (1989), afirma que: "por lo general determinan tendencias, identifican relaciones potenciales entre variables y establecen el "tono" de investigaciones posteriores más rigurosas" (p. 412). Se diseñó una entrevista semiestructurada en la que pudieran expresar lo relacionado al conocimiento de la asignatura y su relación con su futuro desempeño laboral.

La selección fue voluntaria, donde se les explicó la intensión del estudio y el beneficio que tendría tanto para ellos como para la comunidad educativa con la que se relacionan y sus potenciales centros laborales. Para el análisis de los resultados se diseñó una matriz de doble entrada, para comparar las repuestas y encontrar las coincidencias y al mismo tiempo poder elaborar la socialización de las ideas.

\section{Resultados}

Después de organizar las respuestas obtenidas nuestros estudiantes consideran:

Que el dominio de las matemáticas es un arte, que requiere de mucho tiempo, dedicación y requiere del conocimiento. Demanda muchas cosas. Trasmitir el 
conocimiento es más que un arte, esto en relación con la definición de la Didáctica de las Matemáticas.

Además, consideran que, la problemática más relevante en la enseñanza de las matemáticas es la falta de interés en los educandos, lo que hace que el docente se desanime, así como la falta de investigación y búsqueda de nuevos métodos de enseñanza que se adapten a la nueva generación de estudiantes. Así mismo, la resistencia al cambio tecnológico (uso de las TIC). Esto en relación con las problemas y dificultades al enseñar las matemáticas.

En correspondencia a los desafíos y retos para la enseñanza de las matemáticas, la mayoría concuerda en lograr que los estudiantes vean el estudio de las matemáticas con interés e importancia para la vida, otro de los retos es el dominio de competencias pedagógicas y manejo de herramientas tecnológicas, de la cual muchos docentes carecen de ellas, así mismo se necesitan nuevas técnicas de evaluación.

En relación con las características de un proceso de enseñanza tradicional, coincidieron en que, en el aula el aprendizaje es mecanizado, el docente explica el tema, da los teoremas, reglas a utilizar, esperando que los estudiantes solo recepcionan el contenido, piden que los estudiantes se aprendan la cantidad de información para ser usado en nuevos contenidos, explican un par de ejercicios de un nivel medio y brindan otra cantidad de ejercicios con un nivel de mayor dificultad para que los alumnos trabajen el resto del tiempo, explican de manera rápida sin importar que los alumnos aprendan o no, preparar la planificación diaria de acuerdo a conocimientos sin tomar en cuenta la práctica.

En analogía con las estrategias innovadoras para la enseñanza de las matemáticas expresan que, el uso de las TIC, por ejemplo el del celular en los estudiantes es muy difícil de evitar y casi el $100 \%$ de los estudiantes tienen este recurso, y como docentes deberían de revertirlo y convertirlo en una herramienta didáctica, otra está referida al aprendizaje mediante el juego, como por ejemplo cartas de ecuaciones, monopolio de operaciones, el enfoque de resolución de problemas, así como el llevar a la práctica algunos contenidos en el campo que les rodean dentro del centro educativo, tener en cuenta que el contenido sea significativo para el estudiante.

\section{Discusión y conclusiones}

La mayoría de los estudiantes tienen la idea general de que la Didáctica tiene mucho que ver con el "Arte de Enseñar", sin embargo, manifiestan que existen docentes que no tienen ni idea de lo que esta significa, por la forma como imparten la clase.

Una de las grandes dificultades que manifiestan los estudiantes es que los docentes con sus "viejas" formas de enseñar, no son capaces de despertar el interés 
de los estudiantes por la asignatura de Matemática. Uno de los desafíos y retos más señalados es que los docentes de Matemáticas deben "actualizarse", en relación con las nuevas tecnologías, y al cómo utilizarlas en el aula, así como al poco uso de estrategias innovadoras.

Tienen ideas bien fundadas en cuanto a las características de un proceso educativo tradicional, señalando que "en la mayoría de sus años" de estudios lo han experimentado en los distintos niveles educativos. y Finalmente, señalan que las estrategias innovadoras están ligadas al "uso de las TIC", en el aula y que al combinarlas con los trabajos en equipos y juegos se puede tener una enseñanza significativa de las matemáticas.

\section{Lista de referencias}

Brousseau, G. (2007). Iniciación al Estudio de la Teoría de Situaciones Didáctica. Buenos Aires : Zorzal .

Contreras, F. (2012). La Evolución de la Didáctica de las Matemáticas . Horizonte de la Ciencia , 20-25.

Chevallard, Y. (1998). La Transposición Didáctica del Saber Sabio al Saber Enseñado. Madrid: AIQUE.

D'Amore, B. (2005). Bases Filosóficas, Pedagógicas, Epistemológica y Conceptuales de la Didáctica de las Matemáticas . México : REVERTE.

Danhke, G. (1989). Investigación y Comunicación. México : McGraw-Hill.

García, J. (2019). La Didáctica de las Matemáticas: Una Visión General . EDUCREA, 12-19.

Martínez, M. D., \& Romero , T. (2019). Transición de la Aritmética al Álgebra:Un estudio con estuidantes universitarios de Nicaragua. Revista Electrónica de Conocimientos, Saberes y Práticas , 29-39.

Melquiades, A. (12 de Abril de 2014). Estrategias Didácticas para un Aprendizaje Constructivista en la Enseñanza de las Matemáticas. Obtenido de Perspectivas Docentes : file://C:/Users/USUARIO/Downloads/Dialnet-EstrategiasDidact icasParaUnAprendizajeConstructivi-6349169.pdf

OEI. (29 de Agosto de 2018). Iberoamérica Divulga. Obtenido de Incursión de las TIC en la Educación Superior : https://www.oei.es/historico/ divulgacioncientifica/?Incursion-de-las-TIC-en-la-educacion-superior 


\section{INVESTIGACIÓN EDUCATIVA}

RTVE.ES. (o9 de 12 de 2013). "El primer problema con las matemáticas es que no se entiende lo que se lee". Obtenido de https:/www.rtve.es/noticias/20131209/ primer-problema-matematicas-no-se-entiende-se-lee/812561.shtml

Van Arken, H. (18 de Febrero de 2018). Modelos Pedagógicos de la Escuela Tradicional. Obtenido de Pedagogía Docente : https://pedagogiadocente.wordpress.com/ modelos-pedagogicos/la-escuela-tradicional 